\title{
EMPREGO DE TECNOLOGIAS EM LEVANTAMENTO E DIAGNÓSTICO DE EDIFICAÇÕES HISTÓRICAS DE LAGUNA, SC ${ }^{1}$
}

\author{
TECHNOLOGY EMPLOYMENT FOR SURVEY AND DIAGNOSIS OF \\ HISTORICAL BUILDINGS OF LAGUNA, SC
}

\author{
Douglas Heidtmann Junior \\ Universidade do Estado de Santa Catarina, UDESC \\ douglasemerson@gmail.com \\ Maicon Padilha \\ Universidade do Estado de Santa Catarina, UDESC \\ maicon.fedrigo@gmail.com
}

\begin{abstract}
Resumo
Este trabalho apresenta os resultados obtidos com o uso de tecnologias digitais voltadas à fotogrametria terrestre e à modelagem de Informação da Construção (Building Information Modelling - BIM), como método aplicado ao levantamento e diagnóstico de edificações de interesse histórico e cultural. Utilizando-se destas técnicas, demonstra-se que há aspectos positivos e negativos para a otimização dos trabalhos realizados em uma disciplina de graduação em Arquitetura e Urbanismo intitulada Técnicas Retrospectivas. Na poligonal de tombamento do IPHAN, em Laguna, são escolhidas semestralmente edificações para que os alunos de arquitetura e urbanismo demonstrem a aplicação dos conhecimentos adquiridos em sala de aula para o levantamento e diagnóstico. Durante o segundo semestre de 2014, um grupo de alunos optou por empregar a Fotogrametria Terrestre para tomada de dimensões externas e detalhes das edificações e o BIM como ferramenta de manipulação de dados e informações para a representação gráfica e geração dos desenhos técnicos exigidos pelos órgãos responsáveis pela preservação do patrimônio. Os resultados sugerem que a utilização da fotogrametria e do BIM presta-se com eficiência para a representação gráfica nesse tipo de trabalho e é ferramenta essencial com a qual estudantes de graduação em Arquitetura e Urbanismo devem buscar familiarizar-se.
\end{abstract}

Palavras-chave: BIM, Fotogrametria, Preservação do Patrimônio.

\begin{abstract}
This paper presents the results obtained by the use of digital technologies of terrestrial photogrammetry and Building Information Modeling (BIM) as a method applied to the survey and diagnosis of historical buildings. By the employment of these techniques, there are positive and negative aspects for the optimization of this kind of work done in an Architecture and Urbanism course by the students of Technical Retrospective. In the polygonal of IPHAN, in Laguna, historical buildings are chosen and students of Architecture and Urbanism try to demonstrate the application of knowledge acquired in the classroom to survey and diagnosis. During the second half of 2014, a group of students chose to employ the Terrestrial Photogrammetry for making external dimensions and details of buildings and BIM as data manipulation tool and information for graphical representation and generation of technical drawings required by the heritage agencies. The results suggest that the use of Photogrammetry and BIM are
\end{abstract}

\footnotetext{
${ }^{1}$ HEIDTMANN, D.; PADILHA, M. Emprego de tecnologias em levantamento e diagnóstico de edificações históricas de Laguna, SC. In: VII Encontro Nacional de Tecnologia de Informação e Comunicação na Construção - TIC 2015, 2015, Recife. Anais... Porto Alegre: ANTAC, 2015.
} 
effective for graphic representation in this type of work and it is an essential tool with which Architecture and Urbanism students should become familiarized.

Keywords: Building Information Modeling, Photogrammetry, Heritage Preservation

\section{INTRODUÇÃO}

Este artigo resulta da atividade pedagógica em andamento no Curso de Arquitetura e Urbanismo da Universidade do Estado de Santa Catarina (UDESC), sediado no Campus VI, no Centro de Educação Superior da Região Sul (CERES) na cidade de Laguna, Santa Catarina.

Por meio deste artigo propõe-se a reflexão sobre o ensino de projeto em preexistências arquitetônicas protegidas por tombamento, a partir dos resultados obtidos com o uso de tecnologias digitais voltadas à fotogrametria terrestre e à modelagem de Informação da Construção (Building Information Modelling - BIM), como método aplicado ao levantamento e diagnóstico de edificações de interesse histórico e cultural por meio de duas disciplinas relativas ao tema da Preservação do Patrimônio Cultural: Técnicas Retrospectivas: Teoria e Projetos; e Projeto Restauração do Patrimônio Arquitetônico.

Laguna localiza-se no litoral sul catarinense, há cerca de $110 \mathrm{Km}$ de Florianópolis/SC, e constitui a terceira cidade mais antiga do Estado de Santa Catarina, fundada depois de São Francisco do Sul e Desterro, atual Florianópolis. O município de Laguna tem uma poligonal (ver Figura 01) protegida oficialmente por tombamento federal pelo Instituto do Patrimônio Histórico e Artístico Nacional (IPHAN) desde 1985 e conta com Escritório Técnico do IPHAN (ETEC-IPHAN).

Figura 01. Poligonal de Tombamento de Laguna.

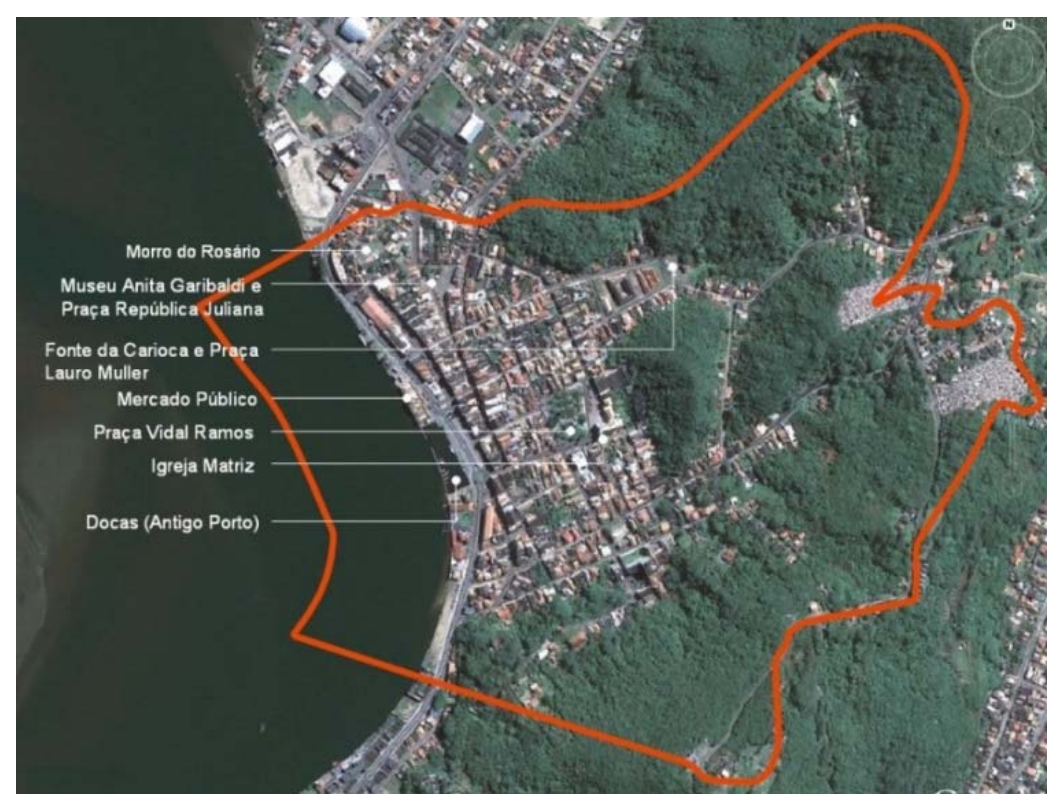

Fonte: Elaborado pelo autor, 2015.

$\mathrm{Na}$ poligonal de tombamento do IPHAN em Laguna, são escolhidas semestralmente edificações para que os alunos do curso de Arquitetura e Urbanismo demonstrem a aplicação dos conhecimentos adquiridos em sala de aula para o levantamento e diagnóstico. Durante o segundo semestre de 2014, um grupo de alunos optou por empregar métodos que, até então, não tinham sido utilizados para documentação arquitetônica (levantamento e diagnostico), na 
disciplina: Fotogrametria Terrestre e Modelagem da Informação da Construção.

\section{OS CONCEITOS FUNDAMENTAIS}

\subsection{Documentação arquitetônica}

A documentação pode ser entendida como apoio à preservação do patrimônio cultural, tendo sido mencionada pela Carta de Veneza, publicada no II Congresso Internacional de Arquitetos em 1964 (FRANÇA et al, 2004). A carta estabeleceu que os trabalhos de conservação e restauro deveriam ser acompanhados de processo de documentação precisa sob a forma de relatórios analíticos e críticos, ilustrados por desenhos e fotografias. A documentação arquitetônica é entendida como o processo sistemático de aquisição, tratamento, indexação, armazenamento, recuperação, disponibilização e divulgação de dados e informações, gráficas e não gráficas, sobre as edificações e os sítios onde estão inseridas, para os mais variados usos (AMORIM, 2007). Os procedimentos envolvidos na documentação arquitetônica passam a ter um papel mais relevante e adquire novos modos com a evolução da tecnologia.

\subsection{Fotogrametria}

A palavra Fotogrametria é derivada de três palavras de origem grega: "photos" (luz), "gramma" (descrição) e "metron" (medida). E pode ser definida como uma técnica de extrair de fotografias, as formas, as dimensões e as posições dos objetos nelas contidos (AMORIM et GROETELAARS, 2004). A Fotogrametria também pode ser definida como a ciência e tecnologia para a determinação de informações precisas de objetos e do meio ambiente, a partir do registro, mensuração e interpretação de imagens fotográficas (LOCH \& LAPOLLI, 1994). Entre os maiores benefícios da Fotogrametria sobre outros procedimentos de campo pode-se citar: o aumento da precisão, a informação da construção mais completa, custos reduzidos, além do tempo reduzido a campo. Estes benefícios são resultados da substituição de medidas de campo por medição de imagens. Isto remove a necessidade do acesso físico em cada ponto que a medida é necessária. A aplicação da Fotogrametria é dividida em dois tipos, a terrestre, fotografias tomadas a partir da superfície terrestre, e a aérea, também chamada de aerofotogrametria, quando câmeras são montadas em aeronaves e tomadas a partir de vôos sobre a área de registro.

No campo da Preservação do Patrimônio, o emprego da Fotogrametria terrestre é essencial visto que permite o registro que acumula conhecimento sobre as edificações ajuda na compreensão de aspectos físicos, materiais, construtivo e seu significado histórico e cultural. Entretanto, seu emprego para levantamento de edificações, visando a tomada de medidas com maior precisão ainda pode ser considerado incipiente o que deve-se, em grande parte, ao desconhecimento dos softwares que são usados em tal campo do conhecimento.

\subsection{BIM - Building Information Modeling}

Embora seja possível remontar a origem da Modelagem da Informação da Construção ou BIM - Building Information Modeling à década 1970 (EASTMAN, 1999), apenas no início deste século sistemas comerciais robustos passaram a ser utilizados de forma integrada para esta finalidade. A modelagem da informação da construção (BIM) envolve grande complexidade pela quantidade de conceitos, ferramentas sofisticadas e processos abertos, que objetivam melhorar os índices de produtividade, qualidade e durabilidade da construção, reduzir desperdício de materiais, mão de obra e outros recursos, aumentar o controle sobre os processos envolvidos no projeto, na construção e na utilização das edificações, dentre outros. 
No Brasil, ainda é preciso que haja um processo mais amplo de difusão e adoção do BIM por empresas e profissionais, para que a modelagem torne-se uma inovação efetiva com benefícios para os vários segmentos envolvidos na construção civil, dentre eles, a área de intervenção em edificações de interesse histórico e cultural.

\section{PROCEDIMENTOS METODOLÓGICOS}

Método das tomadas de medição convencional: A tomada de medidas é a base para o levantamento cadastral das edificações de interesse histórico e cultural que deve ser minucioso e acompanhado de registro fotográfico do estado atual do imóvel. Toda a documentação constitui a base de dados para a elaboração do projeto de intervenção. Grande parte dos profissionais que trabalham com restauro desenvolvem o cadastro de forma manual, fotografando a edificação, medindo-a e esboçando-a, segundo os procedimentos mais corriqueiros, tais como a triangulação e as cotas cumulativas. São feitas medições e anotações para assegurar o maior grau possível de detalhamento. $\mathrm{Na}$ fase seguinte, de representação gráfica técnica. São utilizados, normalmente, ferramentas CAD, o que demonstra o uso ainda limitado de ferramentas computacionais, tanto por parte de profissionais quanto de estudantes. No caso dos trabalhos executados para a disciplina de Técnicas Retrospectivas, segue-se os mesmos procedimentos, com o emprego de trenas, níveis, prumos e croquis.

Método da Fotogrametria terrestre: A primeira etapa do processo compreende a tomada fotográfica da edificação em levantamento. Para isto foi empregada uma câmera com resolução de 8 megapixels. Foram escolhidas várias posições, uma com maior ortogonalidade com o plano principal da parede em levantamento e as demais posições foram com ângulos à esquerda e à direita. Na segunda etapa, utilizou-se o programa ImageModeler® onde foram inseridas as imagens raster com maior nitidez e qualidade, onde estavam bem definidos os pontos de interesse. Em seguida, foram referenciados pontos homólogos nas fotografias, usando-se as ferramentas do programa e adquirido um conjunto de informações, como identificação de patologias e mapa de danos, por meio de software BIM.

Método da Modelagem de Informação da Construção: O Método da Modelagem de Informação da Construção foi empregado como ferramenta de manipulação de dados e informações para a representação gráfica e geração dos desenhos técnicos exigidos pelos órgãos responsáveis pela preservação do patrimônio.

\section{RESULTADOS E ANÁLISES}

Os resultados sugerem que a utilização da Fotogrametria Terrestre e do BIM presta-se com eficiência para a representação gráfica nesse tipo de trabalho e é ferramenta essencial a qual estudantes de graduação em Arquitetura e Urbanismo devem buscar familiarizar-se. As conclusões reconhecem que os produtos do obtidos, bem como o método proposto podem ser utilizados em trabalhos de levantamento e diagnóstico e que facilitam as atividades de ensino de disciplinas voltadas à preservação do patrimônio.

A principal vantagem do uso da Fotogrametria foi a agilidade e a possibilidade de medição em lugares altos e inacessíveis. Através do uso da técnica da fotogrametria a medição torna-se muito ágil, pois o tempo efetivo de medição manual é maior e pode depender de mais de uma pessoa em alguns casos. Após a calibragem das imagens no computador a distância entre quaisquer dois pontos pode ser consultada a qualquer momento e não precisa de visita a campo, problema esse que se encontra pelo método manual. Além disso a medição em lugares altos e de difícil acesso são facilitadas com o uso da técnica. A técnica é feita com base na proporcionalidade e triangulação através das fotos, isso significa que uma medida, ao menos, é necessária para que sejam obtidas as demais. 
As principais desvantagens do uso da Fotogrametria foram: Ângulo nem sempre propício, especificações dos equipamentos podem interferir no erro, contexto urbano pode ser desfavorável a medição (carros, postes e árvores). Um dos problemas do uso desta técnica é que muitas vezes o local não é muito favorável para a captura das imagens, por exemplo: barreiras físicas impedem que o fotógrafo se desloque apropriadamente, árvores, postes, carros e pedestres reduzem a quantidade de informação da construção. Para solucionar o problema pode-se utilizar "miras guias" (ver Figura 02) para acrescentar informação e auxiliar na calibragem das fotografias. Os equipamentos também interferem na qualidade e precisão da fotogrametria, pois a resolução da imagem, campo de visão, quantidade de brilho e borrão podem comprometer o erro. Para este caso a câmera utilizada foi a de um tablet Apple iPad® (quarta geração), mas foram feitos testes com outras câmeras, sendo uma Fujifilm® e a outra Canon®, sem muito êxito graças a complexidade de operação e manuseio.

Figura 02. Miras Guias

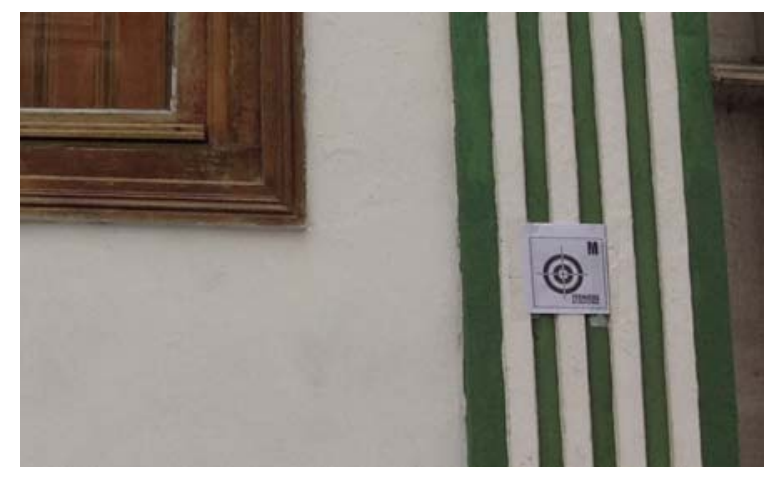

Fonte: Padilha et al., 2015.

Outro fator é que, geralmente, nas partes internas das edificações não há uma boa precisão e necessita-se do auxílio de trena. A fotogrametria do interior dos edifícios não é muito precisa pois necessita de fotos panorâmicas e este tipo de fotografia é difícil de ser obtida com precisão necessária, por isso toda a medição interna e triangulação da planta baixa foi feita pelo método tradicional através de trena. As imagens internas podem ser feitas do modo não panorâmico, mas precisa-se de lentes com campo de visão grande (maiores de 70 graus), estas lentes são muitas vezes caras, mas podem solucionar o problema em alguns casos.

Algumas vantagens foram percebidas quanto à utilização do BIM para o trabalho, tais como:

1. Torna o levantamento mais preciso, pois as imperfeições construtivas ficam evidentes O levantamento de edificações históricas feito em BIM aumenta o nível de precisão pois exige que o levantamento funcione em todos os desenhos simultaneamente - tanto em planta, quanto em fachada ou corte as medições devem encaixar, e caso não aconteça o erro ficará evidente, o que exige que a medição seja refeita.

2. Computação de frequência, volume e quantidade a níveis de tabela - O modelo resultante de BIM traz consigo alguns tipos de informação, entre elas o volume e área de seus elementos. Esse tipo de informação pode ser muito relevante quando se trata de quantificar a área de tinta necessária para pintar as paredes ou forro, ou também, quanto será necessário de material para reconstruir um elemento destruído por exemplo. Existem informações quantitativas, que podem ser usadas para demonstrar quantas peças são repetidas na mesma edificação, o que é algo cansativo muitas vezes. Todas essas informações podem auxiliar nos planos orçamentários de restauração, reforma ou requalificação.

3. Ensaios computacionais, como solar, de vento, entre outros poderão ser feitos neste mesmo modelo, como diferentes propostas para a mesma passagem de tubulação, 
alteração da fachada caso seja retirado algum poste, como é a incidência solar em determinada hora do dia, como é o comportamento do vento - muitos destes ensaios podem interferir na tomada de decisão quando for projetada alguma edificação anexa, ou caso seja feita uma reforma o modelo poderá facilitar a visualização de uma possibilidade nova.

4. Deformidades são representadas e visualizadas nas diversas vistas - As deformações da edificação serão quase sempre representadas, tornando o modelo mais fiel. Essas deformidades são trabalhosas de representar quando utilizado o método em CAD, porém mostram a edificação em sua verdadeira natureza. Portanto, os cortes mostram os elementos muitas vezes inclinados, seguindo rigidamente os princípios de representação de desenho técnico. (ver Figura 03)

5. Facilita a representação (quando levado em conta todas as vistas e perspectivas possíveis até o final do processo), Versatilidade do modelo (explodidas, cortes, esquemas) - As representações gráficas são facilitadas quando pensadas como um todo, pois são todas extraídas de um mesmo modelo - podendo ser feitas perspectivas com ponto de fuga, perspectivas isométricas, cortes, vistas explodidas, elevações internas, elevações externas, cortes perspectivados, cortes perspectivados explodidos, plantas, esquemas etc (ver Figura 04). Embora o tempo de modelagem seja mais demorado, quando comparado com a representação em CAD em uma vista simples, deve-se levar em conta que o mesmo modelo poderá se repetir em diversas vistas e suas alterações são simultâneas a elas.

Figura 03 e 04 . Compoteiras em diferentes profundidades e Perspectiva Isométrica seccionada explodida

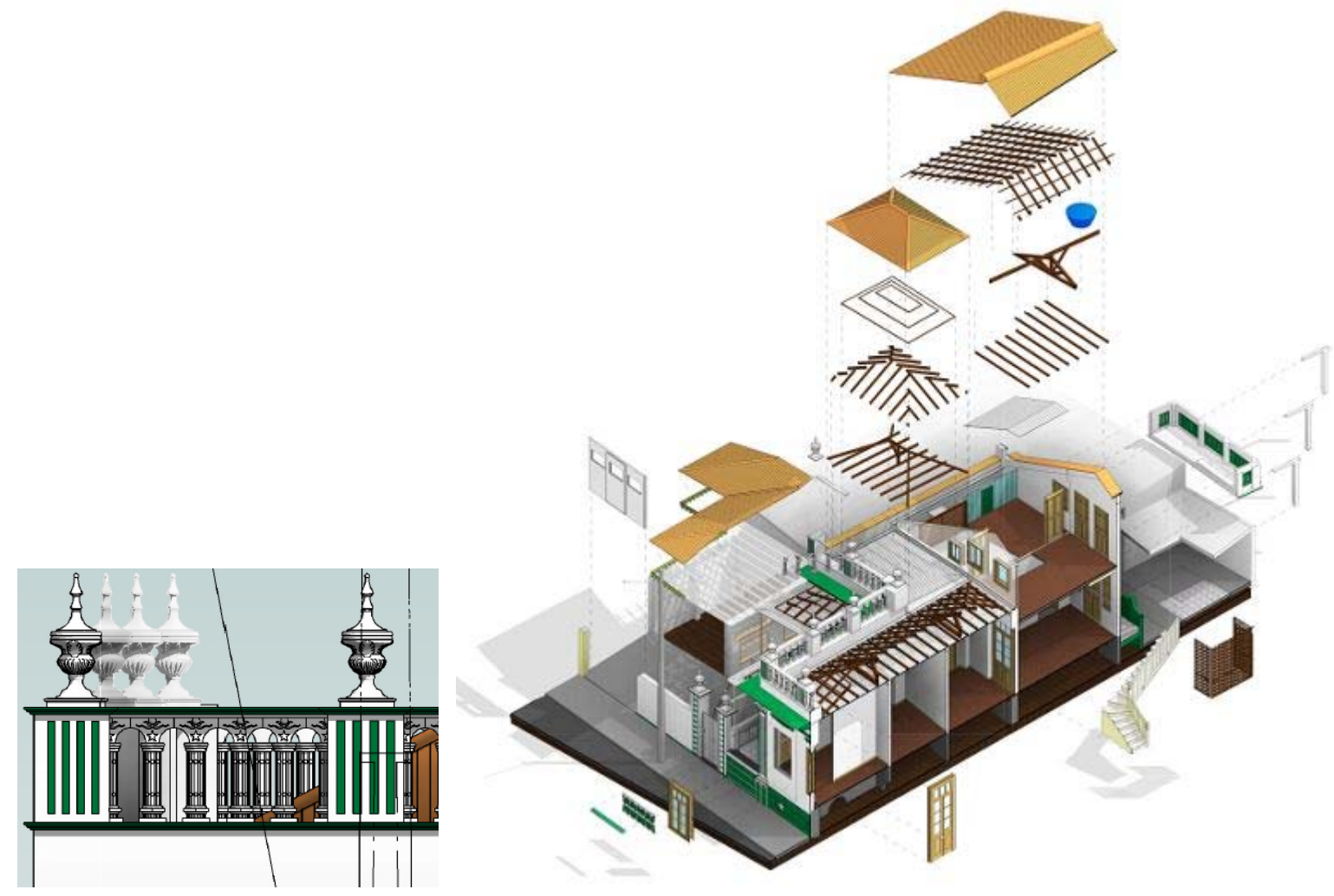

Fonte: Padilha et al., 2015. 
6. Finalização rápida após a conclusão do modelo - A finalização do levantamento é mais rápida quando comparada com o método CAD, pois o uso de templates de visualização poderá mudar automaticamente o modo de apresentação da vistas - levando cor, sombra e cor de fundo por exemplo - o modo como os desenhos são tratados até a impressão final são melhores pois são feitos com a espessura da linha já pré-visualizada durante o processo de modelagem, simultaneamente a isso é possível ver se escala é adequada ao tamanho de papel escolhido.

7. Permite uma integração com amplo suporte para maquete e perspectivas digitais (ver Figura 05), que pode extrapolar em outros campos do conhecimento e em outras artes O modelo criado em BIM pode ser incorporado a outros softwares, como por exemplo o Autodesk Maya e Autodesk 3ds Max, desse modo poderá se utilizar dos recursos destes outros programas, como fazer maquetes digitais e animações, por exemplo: o modelo desta casa foi reconstruído de modo simplificado em cima do modelo BIM para que fosse feito um modelo de papel. (Figura 06)

Figura 05 e 06. Perspectiva com Intervenção e Modelo (Papercraft)

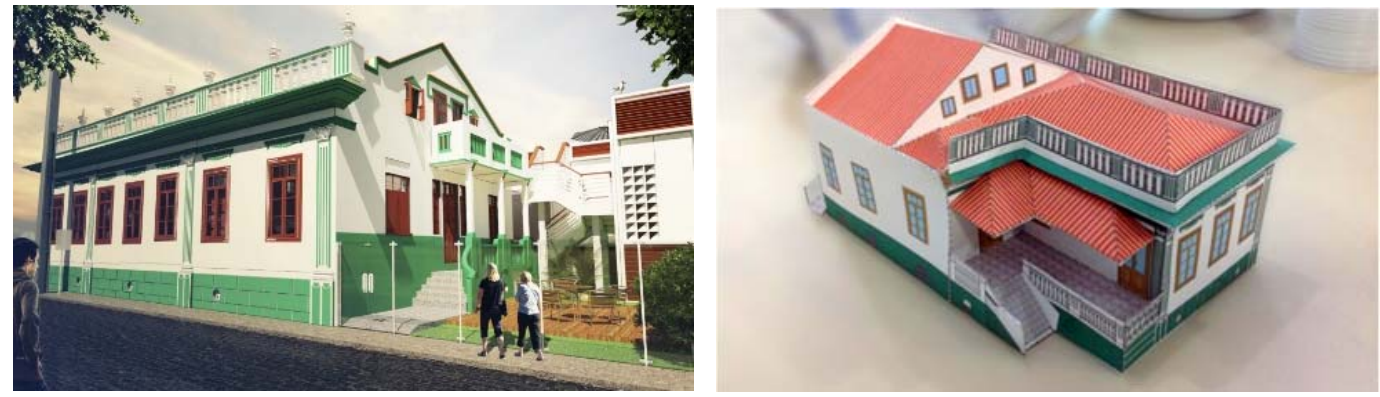

Fonte: Padilha et al., 2015.

8. Representações tendem a ficar mais fiéis pois são automáticas e independem de interpretação humana a princípio.

9. Permite a modelagem simultânea por diversos usuários em um só arquivo - O Processo de modelagem pode ser feito por vários usuários simultaneamente (através de "Worksharing") o que aumenta a produtividade do trabalho em equipe. Durante o processo os usuários podem sincronizar com a central e enviar as suas criações e edições, além de receberem as alterações dos demais usuários. Nessa técnica os membros podem limitar os seus domínios, por exemplo: poderá ser organizado de modo que o "usuário 1" fique somente com o primeiro andar, o "usuário 2" com o segundo e o "usuário 3" com as fachadas. Desse modo ninguém irá alterar o que não for sua responsabilidade. Trabalho em equipe é necessário rede de computadores.

As principais desvantagens percebidas quanto à utilização do BIM para o trabalho, foram:

1. Burocracia maior de início - Quando se inicia uma atividade em BIM parte-se de um template, onde os possíveis elementos, vistas, níveis etc. estão presentes, por isso demanda que as atividades sejam pensadas previamente. Caso não seja possível prever todos os pormenores é possível que durante o processo se perca algum tempo para resolver detalhes, o que não inviabiliza, mas dificulta e demanda tempo.

2. Exigência de um template e views templates - $O$ uso de templates de visualização (View templates) auxilia muito o trabalho, pois evita que o usuário fique alterando todos os parâmetros de visualização como sombras, cores, sol, data, escala, nível de detalhe, elementos visíveis, entre outros. Muitas vezes os elementos que o usuário necessita são específicos, como em uma planta baixa técnica: não demanda de um desenho colorido, 
nem sombras, nem encanamento, em contrapartida precisa de cotas, níveis, janelas e portas. Tendo isso em mente o usuário poderá criar seu Template de Visualização para facilitar seu trabalho.

3. Exige o conhecimento e aplicação dos materiais que serão utilizados

4. Demanda que a interpretação humana seja sempre o mais correta possível, pois pode inviabilizar o modelo inteiro se mal levantada

5. Muito tempo para modelar detalhes e escolher materiais (como cornijas, platibanda, balaústres e compoteiras).

6. Tempo útil modelando é maior do que desenhado (quando levado em conta uma vista somente)

7. Alguns elementos merecem atenção para a redução dos níveis de detalhe, para que seja facilitado o processo de representação e desenho (Figura 07)

Figura 07. Automatização do Nível de Detalhe

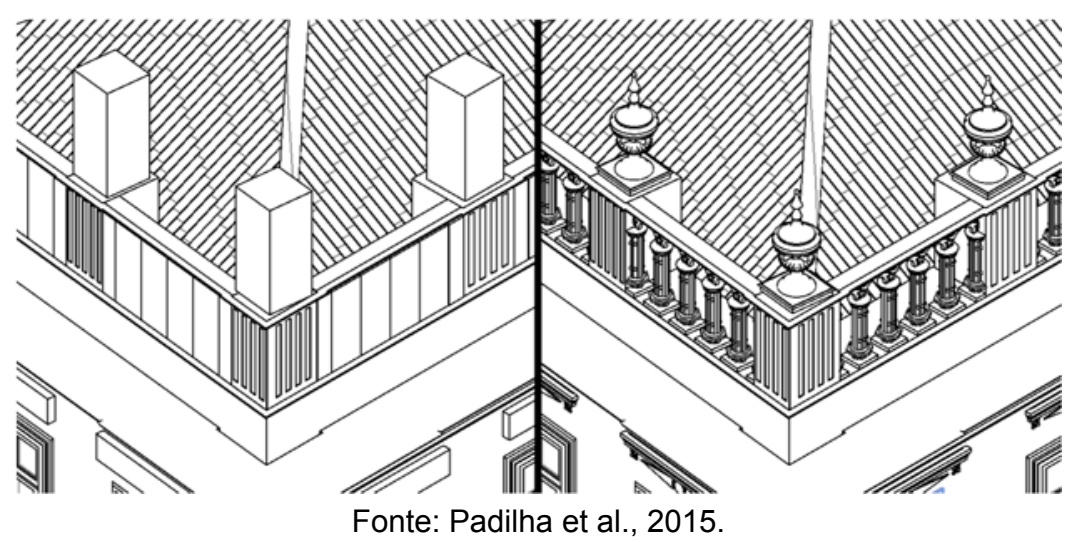

\section{CONCLUSÕES}

Considerando que a experiência ocorreu em âmbito acadêmico, com o objetivo de avaliar a possibilidade de emprego das tecnologias nas disciplinas que tratam de levantamento, diagnostico e projeto de intervenção, chega-se à conclusão de que obteve-se um resultado positivo e que não prejudicou o andamento do trabalho do grupo de alunos.

Quanto à utilização da Fotogrametria, que é caracterizada pela simplicidade, rapidez e baixo custo, considera-se que é plenamente possível sua utilização para a elaboração do Levantamento e Diagnostico de edificações de interesse histórico e cultural, através da seleção das feições de interesse, elementos arquitetônicos e áreas afetadas, que são usadas, por exemplo, como referência para a identificação visual das patologias. Entretanto, ainda é imprescindível, a identificação de patologias in loco, para a caracterização mais efetiva.

Quanto à utilização da Modelagem da Informação da Construção, sua utilização para a representação gráfica do Levantamento, apesar de ter se mostrado interessante, ainda apresenta limitações. O BIM passa a ser de extrema importância na fase do Projeto de Intervenção que, no caso da disciplina envolvida, ocorreu no semestre seguinte e propunha a restauração da edificação existente e o projeto de uma edificação anexa. Através do BIM, pode-se ter o controle de o que será construção nova, o que é existente, temporário e o que será demolido (ver Figura 08). Também podem ser geradas tabelas para cada etapa da obra, por exemplo, quais paredes serão removidas, quais paredes serão acrescentadas, entre outros. Há uma facilidade do ensaio volumétrico, pois possibilita a visualização de diversas possibilidades nas mesmas condições (experimentação), gerando representações quando 
pertencentes às duas partes (existente e nova) que são dinâmicas e não demandam que sejam feitas interpretações através dos cortes e elevações, com uma simples mudança de plano em que a representação é refeita. Torna-se mais fácil a visualização e compreensão das partes simultaneamente, permite-se trabalhar em Fases (Phasing) o que demonstra quais serão as etapas de construção nova.

Figura 08. Construção Nova, Existente e Demolida (Linha Preta, Cinza e Preta Tracejada respectivamente)

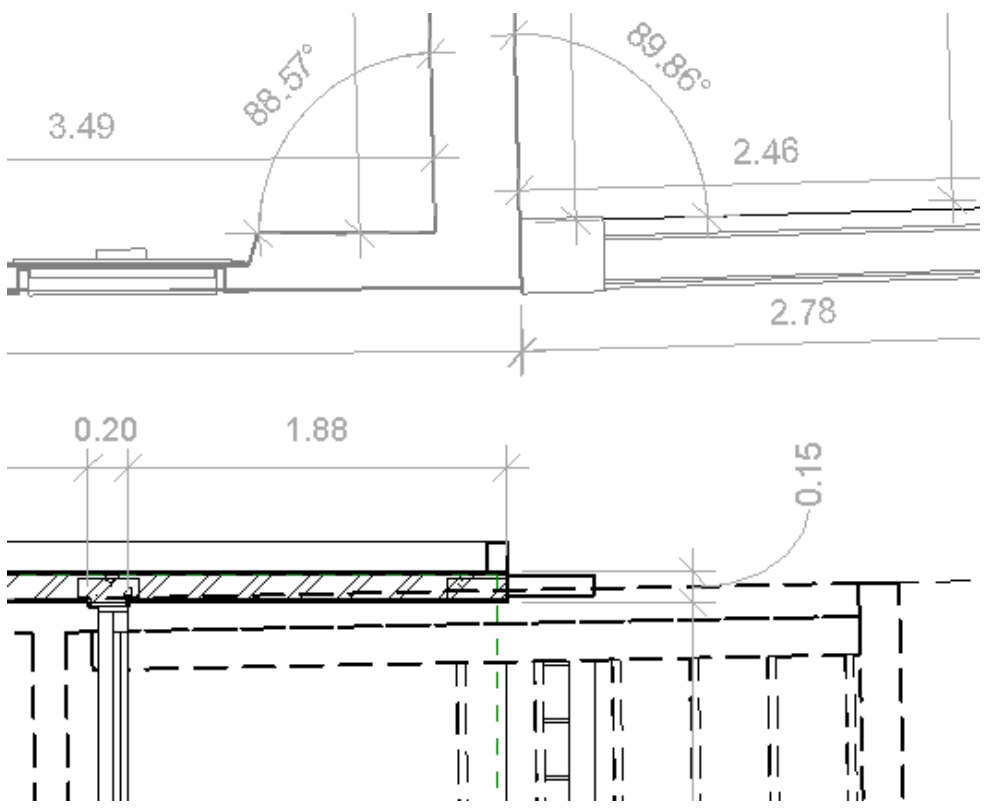

Fonte: Padilha et al., 2015.

Quando comparada com a tecnologia CAD supõe-se que o levantamento se torna possível em detrimento do tamanho da edificação e sua complexidade geométrica, sendo que a gestão do tempo é diferente nas duas técnicas; a CAD permite a conclusão independente dos desenhos e a BIM é simultânea. Finalmente, espera-se com a efetiva incorporação da tecnologia no LABPPAT/UDESC-Laguna o uso da sua potencialidade para documentação arquitetônica mais precisa tais como modelos geométricos tridimensionais e representações ou dados para sistemas de visualização tridimensional com a realidade aumentada.

\section{REFERÊNCIAS}

AMORIM, Arivaldo; CHECCUCCI, Érica. Modelagem da Informação da construção como inovação tecnológica. Anais V TIC - Salvador, Bahia, Brasil, agosto de 2011.

AMORIM, A. L.: 2007, Documenting Architectural Heritage in Bahia - Brazil, Using Digital Technologies. In: XXI International CIPA Symposium, Athens. Disponível em: <http://cipa.icomos.org /fileadmin/papers/Athens2007/FP013.pdf>. Acesso em: 05 de maio de 2008.

EASTMAN, C. M. Building Product Models: Computer Environments Supporting Design and Construction. Florida: CRC Press, 1999. 411 p.

GROETELAARS, N. J. 2004. Um estudo da fotogrametria digital na documentação de formas arquitetônicas e urbanas. Dissertação de Mestrado em Arquitetura e Urbanismo, Universidade Federal da Bahia, Salvador. 
HEIDTMANN JUNIOR, Douglas Emerson Deicke. Novos Usos para Edificações de Interesse Histórico e Cultural: lições da produção arquitetônica pelotense. Florianópolis: 2007. Dissertação (Mestrado em Arquitetura e Urbanismo), CTC-UFSC.

PADILHA, Maicon; BOLDRINI, Leandra; CANDIDO, Juliene; KRETZER, Manuela; PUNDEK, Sofia; TRALDI, Samira. Trabalho de Levantamento e Diagnostico da disciplina Técnicas Retrospectivas, UDESC-Laguna, 2014. Não publicado. 